Revue Française de Civilisation Britannique

XIV-1 | 2006

La dévolution des pouvoirs à l'Écosse et au Pays de Galles 1966-1999

\title{
Les travaillistes et la dévolution 1966-1999
}

Labour and devolution 1966-1999

\section{Carine Berberi}

\section{(2) OpenEdition}

12 Journals

Édition électronique

URL : http://journals.openedition.org/rfcb/1165

DOI : $10.4000 /$ rfcb. 1165

ISSN : 2429-4373

Éditeur

CRECIB - Centre de recherche et d'études en civilisation britannique

Édition imprimée

Date de publication : 2 janvier 2006

Pagination : 69-83

ISBN : 2-911580-23-0

ISSN : 0248-9015

Référence électronique

Carine Berberi, «Les travaillistes et la dévolution 1966-1999 », Revue Française de Civilisation

Britannique [En ligne], XIV-1 | 2006, mis en ligne le 15 octobre 2016, consulté le 20 avril 2019. URL:

http://journals.openedition.org/rfcb/1165; DOI : 10.4000/rfcb.1165

Ce document a été généré automatiquement le 20 avril 2019.

\section{cc) (1) $९$}

Revue française de civilisation britannique est mis à disposition selon les termes de la licence Creative Commons Attribution - Pas d'Utilisation Commerciale - Pas de Modification 4.0 International. 


\title{
Les travaillistes et la dévolution 1966-1999
}

\author{
Labour and devolution 1966-1999
}

\section{Carine Berberi}

1 Depuis sa création, le Parti travailliste a suivi une ligne de conduite peu constante à l'égard des questions relatives à la dévolution. D'abord favorable au début du XX $\mathrm{X}^{\mathrm{e}}$ siècle, comme en témoigna l'engagement de Keir Hardie en faveur de l'autonomie écossaise et galloise, il modifia son point de vue après la Première Guerre mondiale pour deux raisons principales. D'une part, il estimait qu'un gouvernement travailliste ne pourrait mener à bien ses politiques économiques et sociales que par l'intermédiaire d'un gouvernement centralisé, qui assurerait des subventions et aides sociales uniformes dans tout le Royaume-Uni. Des années 1920 jusqu'aux années 1960, le Parti travailliste allait ainsi rejeter toute idée de dévolution en raison de son incompatibilité avec un programme socialiste prônant la planification économique centralisée, et reposant sur le plein emploi et l'État-Providence. D'autre part, les travaillistes craignaient que la dévolution ne réduise leur nombre de sièges en Écosse et au pays de Galles, et ne menace la possibilité pour un gouvernement travailliste d'obtenir une majorité satisfaisante aux Communes. Ainsi, avant 1997, le Parti travailliste ne réussit à obtenir une majorité de sièges en Angleterre qu'à deux reprises, lors des élections législatives de 1945 et de 1966. Lors des autres élections, les voix écossaises et galloises furent essentielles à sa victoire, lui permettant d'obtenir un nombre respectable de sièges à Westminster.

2 Malgré tout, les travaillistes modifièrent leur ligne de conduite sur la question de la dévolution à partir du milieu des années 60. Ils adoptèrent une attitude de plus en plus favorable à la dévolution des pouvoirs à l'Écosse et au pays de Galles, ce qui aboutit finalement à la création du Parlement écossais et de l'Assemblée galloise en 1999. Quels facteurs ont poussé les travaillistes à revoir leur attitude en dépit des incompatibilités que nous avons signalées? 


\section{Les années Wilson et Callaghan (1966-79) : l'échec des deux premiers projets de loi}

\section{Une attitude très prudente en dépit des menaces nationalistes (1966-1970)}

3 C'est à partir du milieu des années 60 que les travaillistes commencèrent à prendre conscience de la montée en puissance des partis nationalistes écossais et gallois à la suite de plusieurs succès électoraux. Au pays de Galles, le Plaid Cymru remporta - aux dépens des travaillistes - l'élection partielle de Carmarthen en juillet 1966 et obtint la deuxième place lors des élections partielles de Rhondda West en mars 1967 et de Caerphilly en juillet 1968. En Écosse, le Scottish National Party (SNP), remporta l'élection législative partielle de Hamilton - bastion travailliste - en novembre 1967, et obtint de très bons scores aux élections locales de 1968. Ces succès ont rapidement inquiété les travaillistes mais ces derniers se trouvaient dans une situation délicate. En raison de l'importance qu'ils attachaient à un programme socialiste reposant sur un État centralisé ainsi qu'aux voix écossaises et galloises pour obtenir une majorité à Westminster, il leur était difficile de soutenir la dévolution. Pour autant, ils ne voulaient pas aliéner un plus grand nombre d'électeurs en refusant toute concession. En décembre 1968, la solution choisie par Harold Wilson, Premier ministre travailliste depuis 1964, fut d'adopter une attitude prudente et d'annoncer la création d'une commission royale sur la constitution (Royal Commission on the Constitution), essentiellement pour gagner du temps ${ }^{1}$. La mise en place de cette commission royale était soutenue par les conservateurs, et notamment par Edward Heath, le dirigeant de l'opposition.

4 Si l'attitude prudente du gouvernement Wilson pouvait s'expliquer par la volonté d'éviter de s'engager en faveur de la dévolution, elle s'expliquait aussi par l'existence d'importantes divisions au sein du Parti travailliste sur cette question. Les ministres du Cabinet étaient partagés quant à l'interprétation des succès électoraux des partis nationalistes qu'il fallait privilégier, certains estimant que la percée nationaliste n'était que passagère, d'autres considérant en revanche qu'elle devait être prise au sérieux ${ }^{2}$. Ces divisions existaient également parmi les députés travaillistes ainsi qu'au niveau des branches écossaise et galloise du parti. Quant au Scottish Council of the Labour Party (SCLP), il avait adopté une attitude très hostile à l'égard de la dévolution dans la mesure où elle nuirait à l'unité du mouvement ouvrier britannique ainsi qu'à toute planification économique centralisée ${ }^{3}$. Les opposants à la dévolution étant très virulents au sein du parti et il était très difficile pour les partisans de se faire entendre. La branche galloise du Parti travailliste, le Welsh Council of Labour, s'était montrée beaucoup plus enthousiaste puisque, dès le milieu des années 1960, elle s'était déclarée favorable à une assemblée élue. Toutefois, tous ses membres ne partageaient pas ce point de vue, particulièrement les travaillistes du sud-est du pays, qui, comme la plupart des travaillistes de l'époque, considéraient que la dévolution risquerait de compromettre les chances du parti d'obtenir une majorité à Westminster . 


\section{L'inaction dans l'attente du rapport Kilbrandon (1970-1974)}

5 La commission royale sur la constitution (Commission Kilbrandon) publia son rapport en octobre 1973 alors que les conservateurs, sous la direction d'Edward Heath, étaient revenus au pouvoir. Si le rapport recommandait majoritairement pour l'Écosse et pour le pays de Galles une dévolution législative, il était plutôt confus et mettait en évidence d'importantes divergences d'opinions, tous les membres de la commission n'étant pas parvenus à se mettre d'accord sur un seul document. Le rapport Kilbrandon fut peu débattu et tomba vite dans l'oubli en raison d'un climat peu propice (premier choc pétrolier, adhésion de la Grande-Bretagne à la Communauté économique européenne...). La réflexion du Parti travailliste britannique sur la question de la dévolution avança donc fort peu au cours de cette période et bon nombre de travaillistes y restèrent opposés. Sans surprise, ils accueillirent assez mal le rapport Kilbrandon, critiquant la confusion de ses recommandations ${ }^{5}$. Le rapport fut également mal accueilli par les branches écossaise et galloise. De nombreux membres du Welsh Council of Labour lui reprochèrent sa confusion et sa complexité. Ils étaient d'autant plus mécontents que, dans le cadre des travaux de la commission, ils avaient clairement fait connaître leur soutien à une assemblée élue ${ }^{6}$. La réaction générale au sein de la branche écossaise fut également négative, de nombreux travaillistes considérant le rapport comme un premier pas vers l'indépendance - même si certains travaillistes écossais, favorables à la mise en place d'un gouvernement dévolu pour freiner la progression du SNP, critiquèrent le minimalisme de ses propositions ${ }^{7}$. Au cours de cette période, un changement commençait à s'opérer au sein du SCLP, certains de ses membres devenant pro-dévolutionnistes. Toutefois, la direction restait, quant à elle, majoritairement hostile, réaffirmant son opposition à une assemblée écossaise à diverses reprises, comme, par exemple, dans Scotland and the UK (1973). Les travaillistes, et les travaillistes écossais notamment, payèrent rapidement le prix de leur ligne de conduite fermement anti-dévolutionniste. En novembre 1973, le SNP remporta la législative partielle de Glasgow-Govan, bastion travailliste des chantiers navals ${ }^{8}$. Ce résultat, très humiliant pour le Parti travailliste alors dans l'opposition, encouragea Harold Wilson à modifier son attitude à l'égard de la dévolution une fois au pouvoir.

\section{L'échec du premier projet de loi en raison d'un parti divisé (1974-1977)}

6 D'abord considérés comme de simples votes de protestation en réaction aux politiques gouvernementales et aux deux grands partis de gouvernement, les succès électoraux de 1967 et 1968 furent confirmés par de nouveaux gains de sièges pour les partis indépendantistes lors des élections législatives de 1974, notamment pour le SNP qui devenait le second parti écossais derrière le Parti travailliste. Ces résultats, qui furent perçus comme une forte montée des nationalismes en Écosse et au pays de Galles, alarmèrent les travaillistes. Le gouvernement Wilson décida donc très vite de faire de la dévolution une priorité, annonçant, dès mars 1974, qu'il ferait rapidement des propositions concrètes pour mettre en œuvre le rapport Kilbrandon. Cet engagement était d'autant plus important à sa survie que le gouvernement, minoritaire entre février et octobre 1974, dépendait de la bonne volonté des nationalistes et des libéraux, favorables à la dévolution. 
7 Dans cette perspective, Wilson chercha à rétablir l'unité au sein de son parti et notamment à convaincre le SCLP. Après avoir exercé diverses pressions sur ce dernier et imposé une convocation à un congrès extraordinaire en août 1974, le SCLP consentit à soutenir le principe d'une assemblée écossaise élue au suffrage direct et disposant de pouvoirs législatifs. Le Parti travailliste réussit à persuader le Welsh Council of Labour de soutenir la proposition d'une assemblée élue avec des pouvoirs non pas législatifs, mais exécutifs' ${ }^{9}$. Dès lors, le gouvernement travailliste put accélérer son programme de réforme en publiant plusieurs documents et en faisant de la dévolution la politique officielle du parti. En novembre 1975, un livre blanc intitulé Our Changing Democracy: Devolution to Scotland and Wales indiquait clairement les intentions du gouvernement et l'engageait sur un certain nombre de principes qui allaient constituer les bases de la loi de 1977: des assemblées élues au suffrage direct seraient créées en Écosse et au pays de Galles; l'assemblée écossaise aurait des fonctions législatives alors que l'assemblée galloise aurait des fonctions exécutives ; ces assemblées seraient élues au scrutin majoritaire (first-pastthe-post system); financées principalement par une subvention globale (block grant) accordée par le Parlement chaque année, elles ne disposeraient d'aucun pouvoir fiscal ; le nombre de députés écossais et gallois siégeant à Westminster ne serait pas réduit; les secrétaires d'État pour l'Écosse et le pays de Galles seraient maintenus et continueraient de siéger au Cabinet de Londres. Toutefois, deux de ses propositions provoquèrent de nombreuses protestations en Écosse et au pays de Galles: d'une part, les pouvoirs des nouvelles assemblées étaient très limités, aucun transfert de pouvoir n'étant prévu pour l'industrie, l'énergie et l'agriculture dans le domaine économique; d'autre part, le secrétaire d'État conservait d'importants pouvoirs de tutelle sur l'assemblée et sur l'exécutif qui en émanerait. Cette conception minimaliste de la dévolution engendra également des divisions parmi les travaillistes, suscitant notamment le départ de deux de ses membres, Jim Sillars et John Robertson, qui décidèrent de créer le Scottish Labour Party ${ }^{10}$.

8 En dépit de ces critiques, le livre blanc de 1975 servit de base au projet de loi sur la dévolution (Scotland and Wales Bill), présenté par le gouvernement Callaghan aux Communes en $1976^{11}$. Confronté à une forte opposition, notamment de la part des antidévolutionnistes travaillistes, le projet de loi dut finalement être retiré le 22 février 1977 après trois mois de débats à Westminster. C'était la première fois depuis 1969 qu'un projet de loi important proposé par le gouvernement était rejeté par la Chambre des communes.

Il faut dire que beaucoup trop de divisions subsistaient dans les rangs travaillistes en dépit des efforts faits par la direction pour unifier le parti ${ }^{12}$. À Londres, le groupe parlementaire était très divisé. Tout au long de ces années, bon nombre de travaillistes avaient continué à exprimer leur opposition à la dévolution, mettant en avant son incompatibilité avec la mise en place d'un programme socialiste ou les conflits qui pourraient apparaître entre ces nouvelles Assemblées et Westminster. Les députés travaillistes représentant les régions situées au nord de l'Angleterre étaient particulièrement virulents, craignant que la dévolution ne fasse profiter l'Écosse et le pays de Galles d'un traitement de faveur, d'aides régionales plus élevées, comparé à leurs régions ${ }^{13}$. Ils soulignaient également l'injustice suivante : après la dévolution, les députés écossais et gallois à Westminster pourraient continuer à voter sur les problèmes n'affectant que l'Angleterre alors que les parlementaires anglais ne pourraient plus intervenir sur les questions relatives à l'Écosse et au pays de Galles (West Lothian Question) ${ }^{14}$. Face à une telle opposition, les partisans de la dévolution, minoritaires, avaient du mal 
à se faire entendre. Même le Cabinet n'était pas exempt de tensions. Si Harold Wilson dans un premier temps, puis James Callaghan, étaient déterminés à changer la politique du parti en matière de dévolution, tous les membres n'étaient pas unanimes. La majorité semblait même plutôt sceptique, voire opposée, craignant essentiellement que la dévolution n'ouvre la porte au séparatisme et qu'elle n'entraîne une réduction du nombre des députés écossais et gallois à Westminster ${ }^{15}$.

Des tensions persistaient également au niveau de l'Écosse et du pays de Galles. La branche écossaise restait divisée même si son exécutif abandonnait progressivement ses réticences à l'égard de la dévolution, adoptant une attitude plus nuancée et acceptant une dévolution «minimaliste » afin de contrer les menaces nationalistes. En 1976, il était devenu plus favorable à la dévolution ${ }^{16}$. Quant à la branche galloise, elle avait réussi à maintenir son point de vue pro-dévolutionniste, faisant campagne lors des deux élections de 1974 pour une Assemblée élue. Toutefois, un petit groupe mené par six députés travaillistes du sud du pays de Galles continuait à s'opposer à cette ligne de conduite. Incluant Leo Abse (Pontypool), Neil Kinnock (Bedwellty), Donald Anderson (Swansea East), Fred Evans (Caerphilly), Ioan Evans (Aberdare), Ifor Davies (Gower), ce groupe affirmait que la dévolution aboutirait inévitablement à l'indépendance, à la négation de la planification centralisée (base du socialisme), qu'elle menacerait l'unité de la classe ouvrière ainsi que les subventions régionales octroyées au pays de Galles via Westminster ${ }^{17}$. La publication du projet de loi divisa donc le Labour Party in Wales ${ }^{18}$.

\section{Un deuxième projet de loi qui se heurte aux mêmes obstacles (1977-1979)}

11 Après l'échec du premier projet de loi, le gouvernement Callaghan, minoritaire depuis février 1977, fut contraint de signer un pacte de soutien parlementaire avec le Parti libéral (Lib-Lab Pact) afin d'assurer sa survie. En contrepartie, les libéraux demandèrent la réintroduction d'un nouveau projet de dévolution, insistant sur deux exigences: l'attribution de pouvoirs fiscaux spécifiques aux Assemblées, et leur élection à la représentation proportionnelle. Le gouvernement travailliste promit de les satisfaire, mais sans y parvenir. En novembre 1977, le gouvernement Callaghan présenta cette foisci deux projets de loi - un pour l'Écosse (Scotland Bill), un pour le pays de Galles (Wales Bill ) - fondés sur les mêmes principes que le projet précédent. Le gouvernement se trouvait dans une situation délicate et une nouvelle défaite risquait de provoquer une crise politique, voire une dissolution du Parlement, suivie d'élections législatives. En fait, ces projets allaient se heurter aux mêmes obstacles que le précédent projet de loi.

12 Ainsi, ils provoquèrent toujours de nombreuses discussions et divisions au sein du parti même si le gouvernement avait exigé et obtenu une meilleure discipline de vote de la part de ses députés ${ }^{19}$. Certes, il parvint à faire voter le deuxième projet de loi en seconde lecture, puis en troisième lecture au cours du premier semestre de l'année 1978, mais cette victoire ne fut pas remportée facilement. Premièrement, elle fut obtenue au prix d'un marathon parlementaire qui occupa seize jours de débat aux Communes et presque autant aux Lords. Deuxièmement, elle mit fin à une longue guerre de positions principalement animée par deux députés travaillistes écossais : John Smith qui s'était fait l'ardent défenseur du projet, et Tam Dalyell, un infatigable anti-dévolutionniste. Troisièmement, elle fut remportée au prix d'un certain nombre de concessions faites aux travaillistes anti-dévolutionnistes, essentiellement écossais. Ainsi, le gouvernement avait 
dû accepter deux amendements qui allaient lui être néfastes : le premier (présenté par le député travailliste George Cunningham, d'origine écossaise) selon lequel le Scotland Act et le Wales Act ne pourraient être considérés comme définitivement adoptés que s'ils étaient approuvés par au moins $40 \%$ des électeurs inscrits lors d'un référendum préliminaire; le second (dû à Tam Dalyell) selon lequel les référendums ne pourraient être organisés moins de trois mois après une élection générale. Le Scotland Act et le Wales Act reçurent finalement l'assentiment royal le 31 juillet 1978. Ils étaient assez complexes et confus, notamment en sur le partage des responsabilités.

Les deux référendums furent organisés le $1^{\text {er }}$ mars 1979 dans un contexte politique peu favorable au gouvernement Callaghan puisqu'il souffrait d'une baisse de popularité à la suite des grèves à répétition de l'«hiver du mécontentement». Par ailleurs, les campagnes furent marquées par une grande confusion et par de très nombreuses divisions au sein du Parti travailliste comme du Parti conservateur. En Écosse, le camp du « oui » se composait de la Labour Movement Yes Campaign, incluant des éléments de l'aile gauche et de l'aile droite du Parti travailliste; du Yes for Scotland, dominé par les nationalistes mais comprenant aussi des représentants de la société civile et certains travaillistes ${ }^{20}$; et de Alliance for an Assembly, organisation plus marginale et animée par quelques conservateurs et libéraux écossais. Le camp du « non », lui, était mieux organisé, constitué de la Labour says 'No' campaign, menée par Tam Dalyell et Robin Cook, et de Scotland says No, regroupant les conservateurs et les milieux d'affaires. Le camp du «non » était également plus uni dans la mesure où tous ses membres utilisaient les mêmes arguments contre la dévolution, ce qui n'était pas le cas du camp du « oui » au sein duquel travaillistes et nationalistes recommandaient la dévolution pour des raisons diamétralement opposées ${ }^{21}$. Au pays de Galles, des divisions similaires étaient perceptibles puisque le camp du « oui » regroupait l'organisation transpartisane Yes to the Assembly Campaign, le Plaid Cymru, et les travaillistes, qui lancèrent leur propre croisade pour essayer de persuader l'électorat des avantages d'une Assemblée galloise. Ici aussi le débat fut dominé par le camp du «non", sous l'influence des conservateurs gallois, de l'organisation transpartisane No to the Assembly Campaign, et du petit groupe de députés travaillistes gallois dissidents mené par Abse et Kinnock. Les opposants étaient encore une fois unis, notamment dans les critiques qu'ils formulaient à l'encontre de l'Assemblée ${ }^{22}$.

14 Ces référendums furent un lourde défaite pour le gouvernement: en Écosse une petite majorité - 51,6\% des votants, mais 32,9\% des électeurs - vota en faveur du Scotland Act ( $48,4 \%$ des votants votèrent contre) ; au pays de Galles $20,2 \%$ des votants et $11,8 \%$ des électeurs soutinrent le Wales Act (79,8\% des votants s'y opposèrent). Au pays de Galles, ce rejet, massif, représentait un échec total pour le gouvernement travailliste. En Écosse, une majorité de votants avaient certes soutenu le projet de loi, mais le pourcentage en termes d'électeurs était insuffisant puisqu'il ne franchissait pas la barre des $40 \%$. Par ailleurs, la participation avait été assez faible, $63,7 \%$ des électeurs en Écosse et 58,3\% au pays de Galles. À la suite de cet échec, le gouvernement tenta de maintenir le projet de dévolution, surtout en Écosse, mais il n'y parvint pas. Il fut renversé le 28 mars 1979 à la suite d'une motion de censure. Les nouvelles élections législatives, organisées en mai 1979, virent la victoire de Margaret Thatcher, qui abrogea le Scotland Act et le Wales Act en juin 1979. 


\section{Les années Foot, Kinnock et Smith : un engagement renforcé en faveur de la dévolution (1979-1994)}

\section{Le maintien de la dévolution en dépit de l'AES (1979-1983)}

15 Au cours de cette période, la question de la dévolution fut un peu mise de côté. Tout d'abord, les partis nationalistes reculèrent nettement lors des élections législatives de 1979. De plus, les dirigeants du Parti travailliste estimaient que la dévolution n'était plus à l'ordre du jour ${ }^{23}$. Il faut dire qu'ils étaient préoccupés par d'autres problèmes puisque le parti traversait une période difficile, marquée par une phase de remise en question à la suite de la lourde défaite de 1979, par diverses restructurations internes ${ }^{24}$ et par une montée en puissance de la gauche, illustrée par l'élection de Michael Foot à sa tête en 1980. Par ailleurs, l'adoption d'une stratégie économique alternative (Alternative Economic Strategy - AES) lors du Congrès du parti de 1980 ne facilitait pas le soutien à la dévolution. Cette stratégie impliquait effectivement un État très centralisé puisqu'elle avait pour éléments majeurs une politique d'expansion cherchant à restaurer le plein emploi, la multiplication des nationalisations, la planification économique, le contrôle de l'inflation, l'imposition de contrôles sur le commerce international et le retrait de la CEE.

16 Pour autant, le Parti travailliste ne devint pas anti-dévolutionniste et continua à soutenir une Assemblée écossaise, ce qui était assez paradoxal. Dans son manifeste électoral de 1983, il favorisait même une Assemblée disposant de pouvoirs en matière fiscale ${ }^{25}$. L'opposition AES / dévolution ne fut résolue que peu de temps avant les élections législatives de 1983, lorsque les travaillistes définirent une "stratégie régionale alternative " (Alternative Regional Strategy) où l'Écosse était incluse dans le cadre d'un système britannique de planification à la fois national et régional. Il était affirmé qu'un gouvernement dévolu écossais contribuerait à la mise en place d'un programme de croissance coordonnée puisqu'il serait une institution démocratique disposée, et apte, à suivre une telle politique économique interventionniste ${ }^{26}$. Le maintien de ce point de vue avait sans doute été motivé par la branche écossaise du Parti travailliste qui avait adopté une attitude très clairement pro-dévolutionniste peu de temps après les élections législatives de 1979. Elle réaffirma son soutien à un gouvernement dévolu et s'engagea même rapidement en faveur d'une Assemblée disposant de pouvoirs fiscaux, sous l'influence d'un exécutif qui n'allait pratiquement plus jamais exprimer son opposition au principe de dévolution ${ }^{27}$.

17 À partir de 1983, la dévolution des pouvoirs à l'Écosse reprit toute son importance dans les discussions internes au Parti travailliste. L'idée d'un gouvernement dévolu au pays de Galles fut en revanche abandonnée. Il fallut attendre la fin des années 80 pour que les travaillistes renouvellent cette proposition.

\section{Retour de la dévolution au premier plan face à une frustration croissante (1983-1992)}

Les années Kinnock furent marquées par trois défaites électorales successives (en 1983, 1987 et 1992), en dépit d'un processus de modernisation initié par Neil Kinnock après la défaite de 1983 afin de rendre le parti de nouveau éligible en améliorant son image - en éliminant notamment toute connotation gauchiste / extrémiste-, et en rendant ses 
politiques plus populaires et attrayantes. En 1987, les travaillistes complétèrent ce premier travail de modernisation par un nouveau programme de réformes, intitulé Policy Review. Dans ce cadre, les politiques expansionnistes, du type de l'AES, furent abandonnées et les travaillistes commencèrent à accepter le rôle du marché.

Ces défaites eurent également des conséquences sur la politique suivie par le parti à l'égard de la dévolution puisqu'elles relancèrent la question d'un gouvernement dévolu en Écosse au sein du Parti travailliste. L'abandon de l'AES facilitait le soutien du parti à la dévolution et les travaillistes restaient le parti dominant en Écosse en dépit de leur défaite en Angleterre. Afin de conserver le soutien de leurs sympathisants en Écosse, ils décidèrent de s'engager définitivement en faveur d'une Assemblée écossaise dans le cadre d'un Scotland Act amélioré, lançant une campagne de promotion de la dévolution à l'intérieur du parti afin de convertir essentiellement les travaillistes anglais à cette idée. Cette approche allait progressivement être soutenue par la majorité des travaillistes et travaillistes écossais ${ }^{28}$. Neil Kinnock refléta parfaitement ce nouvel état d'esprit puisqu'il soutenait désormais une Assemblée écossaise élue.

Cette nouvelle ligne de conduite était encouragée par un sentiment de mécontentement qui se développait alors en Écosse à l'encontre des gouvernements Thatcher. Ce dernier atteignit son paroxysme en 1989 lorsque les conservateurs décidèrent de mettre en place, en Écosse, un nouvel impôt local, la poll tax, un an avant de l'appliquer à l'Angleterre et au pays de Galles. En signe de protestation, une campagne, menée par le SNP et certains groupes travaillistes dissidents, fut lancée afin de boycotter le paiement de la poll tax et de contraindre les conservateurs à retirer cet impôt. Les travaillistes écossais n'en tirèrent toutefois pas profit puisqu'ils refusèrent d'y participer. De manière générale, ils étaient plutôt passifs, conscients qu'ils ne pouvaient pas empêcher le vote d'une législation hostile à l'Écosse par la majorité conservatrice du Parlement. Cette paralysie leur porta préjudice puisqu'elle expliqua, en partie, la défaite du Parti travailliste face au SNP, lors de l'élection partielle de Glasgow-Govan en novembre 1988. Néanmoins, cette défaite eut des conséquences positives puisqu'elle persuada finalement le parti de réagir afin de ne pas perdre le soutien de ses sympathisants ${ }^{29}$. Les travaillistes écossais allaient désormais prendre une part active à la réunion d'une Convention constitutionnelle écossaise ( Scottish Constitutional Convention) dont l'idée venait d'être lancée par le mouvement Campaign for a Scottish Assembly (CSA) ${ }^{30}$.

21 Cette Convention constitutionnelle visait à réfléchir sur les modalités de mise en place d'un gouvernement dévolu en Écosse. Elle réunissait des personnes de divers horizons : hommes et femmes politiques issus de tous les partis (à l'exception des conservateurs et du SNP), des représentants des administrations locales, des syndicats, des églises, des PME, ainsi que des associations féministes et représentatives des minorités ethniques. Son premier rapport Towards Scotland's Parliament. A Report to the People, publié en 1990, reflétait les compromis passés par ces différents membres, et notamment par les travaillistes écossais. En dépit de leur domination quantitative dans les groupes de travail, ces derniers s'étaient mis à l'écoute de leurs partenaires et avaient fait quatre concessions: ils acceptaient la création d'un Parlement écossais et non plus d'une Assemblée ; ils favorisaient l'idée d'une représentation égalitaire des femmes au sein du futur Parlement; ils acceptaient que le Parlement soit doté de pouvoirs fiscaux spécifiques; ayant renoncé à l'idée d'un système majoritaire uninominal à un tour, ils envisageaient désormais une forme de représentation proportionnelle (un scrutin «à membre additionnel $)^{31}$. Cette dernière concession était importante dans la mesure où 
elle réduisait énormément la possibilité pour le Parti travailliste de dominer le futur Parlement écossais, en accroissant les probabilités d'une coalition.

Au cours de cette période, la question de la dévolution avait également été remise au premier plan au pays de Galles où, de manière similaire à l'Écosse, un mécontentement croissant s'était manifesté à l'encontre des gouvernements conservateurs - d'autant plus que le Parti travailliste y était resté le parti dominant. Ici aussi les travaillistes commencèrent donc à remettre en question la légitimité d'un gouvernement conservateur et à mener des réflexions sur la dévolution. Néanmoins, contrairement à la branche écossaise, la branche galloise du Parti travailliste refusa de coopérer avec les autres partis sur cette question. Ainsi elle refusa de participer à l'idée d'une Convention constitutionnelle galloise proposée par la Campaign for a Welsh Assembly ${ }^{32}$, groupe de pression qui avait été réactivé en 1987. Par ailleurs, elle semblait avoir une vision assez limitée d'une Assemblée galloise, la considérant plutôt comme « un conseil régional » qui serait mis en place au moment de la réorganisation du gouvernement local et qui serait susceptible de remplacer le ministère des Affaires galloises (Welsh Office) et ses quangos ${ }^{33}$.

Au début des années 1990, les documents publiés par le Parti travailliste reflétaient cette période de réflexion et ces avancées en matière de dévolution. Dans Looking to the Future, rapport publié en 1990 dans le cadre de la Policy Review, les travaillistes s'engageaient à créer un Parlement écossais doté de pouvoirs fiscaux lors de la première année de leur législature $\mathrm{s}^{34}$. Pour la première fois, la direction travailliste avait donc accepté les concessions faites par les travaillistes écossais et avait fait siennes les propositions de la Convention. Dans son manifeste électoral de 1992, ils précisaient également qu'une Assemblée galloise serait créée au cours de la législature d'un gouvernement travailliste même si ses pouvoirs étaient définis moins clairement ${ }^{35}$. À partir de la fin des années 1980, les travaillistes commencèrent à considérer la dévolution comme un moyen de mettre un terme à la domination des gouvernements conservateurs, minoritaires en Écosse et au pays de Galles. Cette évolution fut possible grâce à un durcissement du soutien à la dévolution au sein du Parti travailliste, à tous les niveaux.

\section{La même dévolution pour l'Écosse et le pays de Galles (1992-1994)}

24 À la suite des élections législatives de 1992, qui avaient vu à nouveau la victoire des conservateurs $^{36}$, John Smith succéda à Neil Kinnock à la tête du Parti travailliste. Smith était devenu un farouche défenseur de la dévolution à laquelle il donna un second souffle. Deux semaines seulement après la défaite, il réaffirma l'engagement du parti en faveur d'un Parlement écossais ainsi que sa volonté de concrétiser les propositions de la Convention constitutionnelle ${ }^{37}$. Il semblait également prêt à offrir le même type de dévolution au pays de Galles, à savoir un Parlement doté de pouvoirs législatifs ${ }^{38}$. Pour John Smith, la dévolution faisait partie d'un vaste programme de réformes constitutionnelles affectant tout le Royaume-Uni et visant à mettre un terme à la trop grande centralisation des pouvoirs qui s'était opérée depuis 1979. Ses propositions, définies en 1993, incluaient par exemple (outre la dévolution): l'adoption de la Convention européenne des droits de l'homme, la réforme de la Chambre des Lords et l'établissement d'une législation sur la liberté de l'information ${ }^{39}$. Parallèlement, les travaillistes écossais et John Smith, continuèrent à s'impliquer dans la Convention constitutionnelle qui reprit ses travaux en 1993 après une période de silence due à la frustration engendrée par la défaite de 1992. 
$\mathrm{Au}$ cours de cette période, les divisions existant au sein de la branche écossaise du Parti travailliste, rebaptisée Scottish Labour Party, s'étaient atténuées, grâce notamment à l'assagissement des groupes travaillistes dissidents ${ }^{40}$. Il n'en était pas de même au sein de la branche galloise, dont la direction continuait à refuser de s'impliquer dans des groupes de pression extérieurs au Parti travailliste. En 1994, elle décida finalement de créer sa propre commission, la Constitutional Policy Commission, afin de réfléchir aux modalités de mise en place d'une assemblée galloise. Cette ligne de conduite très prudente, motivée notamment par la crainte d'un échec similaire à celui de 1979, suscita diverses tensions. Alors que certains de ses membres, pro-dévolutionnistes, tentèrent de stimuler un changement, tels des députés ou le nouveau secrétaire d'État à l'Écosse, Ron Davies, d'autres se démarquèrent un peu, en participant à la Campaign for a Welsh Assembly, rebaptisée The Parliament for Wales Campaign en 1993, ainsi qu'au Congrès constitutionnel qu'elle organisa en mars 1994. Ce Congrès se prononça en faveur d'un Parlement élu selon un scrutin proportionnel et disposant de pouvoirs législatifs et fiscaux, ce qui allait bien au-delà de ce que la direction était prête à accepter ${ }^{41}$.

\section{Le New Labour et la dévolution (1994-1999)}

\section{Un parti qui impose ses volontés (1994-1997)}

Le décès brutal de John Smith en mai 1994 ne remit pas en question l'orientation prise par le parti. Tony Blair, le nouveau leader, poursuivit dans la même direction que son prédécesseur: il compléta le processus de transformation du parti, en lui donnant notamment un nouveau nom, New Labour, et conserva la même politique prodévolutionniste même s'il faisait preuve de moins d'enthousiasme. Dès juillet 1994, il réaffirma la promesse faite par le parti d'introduire des gouvernements dévolus en Écosse et au pays de Galles au cours de la première législature d'un gouvernement travailliste. Dans le prolongement de John Smith, il inclut également la dévolution dans un programme de réformes constitutionnelles visant à moderniser et démocratiser l'État britannique ${ }^{42}$. Toutefois, contrairement à John Smith, il ne souhaitait pas offrir le même type de dévolution à l'Écosse et au pays de Galles ${ }^{43}$.

Blair continua également à soutenir les propositions de la Convention constitutionnelle écossaise. Le 30 novembre 1995, cette dernière publia son deuxième rapport, intitulé Scotland's Parliament, Scotland's Right. Il apporta peu de changements au premier document, si ce n'est sur deux points principaux. D'une part, le Parlement compterait désormais 129 membres, dont 73 élus au scrutin majoritaire et 56 élus à la proportionnelle selon le système de membre additionnel. D'autre part, concernant la gestion des ressources, un certain recul s'était opéré puisque le Parlement n'aurait la possibilité d'augmenter ses ressources qu'en faisant varier le taux de base de l'impôt sur le revenu voté à Westminster dans une marge de $3 \%$. Une nouvelle fois, le Parti travailliste fit siennes ces avancées que les conservateurs ne tardèrent pas à dénoncer en concentrant leurs critiques sur les pouvoirs fiscaux, qualifiés de 'tartan tax'. Ces attaques incitèrent la direction travailliste britannique à prendre de nouvelles mesures et donc l'initiative en matière de dévolution ${ }^{44}$. En juin 1996, elle annonça un certain nombre de changements. Premièrement, un référendum serait organisé. Il comporterait deux questions séparées : l'une porterait sur la création d'un Parlement écossais, l'autre sur les pouvoirs fiscaux dont il disposerait. Deuxièmement, le référendum aurait lieu avant que 
la législation ne soit votée par le Parlement. Troisièmement, la règle de la majorité simple (et non celle des $40 \%$ ) s'appliquerait lors de ce référendum.

Au pays de Galles, l'initiative en matière de dévolution passa également assez rapidement entre les mains du Parti travailliste britannique. À partir de 1995, il commença à intervenir dans le processus de prise de décision sur la dévolution, intervention facilitée par le fait que la Commission, mise en place par le Labour Party in Wales, n'avait pas encore fait part de ses propositions ${ }^{45}$. Le rapport intitulé Shaping the Vision qu'elle publia en 1995 fut donc très prudent. Il recommandait simplement la mise en place d'une Assemblée galloise, élue au scrutin majoritaire, assumant les fonctions du Welsh Office et des quangos, mais restant financée par Westminster. Shaping The Vision fut approuvé par Tony Blair, qui décida de faire de ce rapport la base de la législation qui serait introduite au cours de la première année de législature d'un gouvernement travailliste. Plusieurs mesures complémentaires furent annoncées au mois de juin 1996 par Ron Davies : une partie des membres de l'Assemblée galloise serait élue selon un système de représentation proportionnelle, et un référendum serait organisé ${ }^{46}$. Cette nouvelle politique n'était pas soutenue par tous les membres du parti, les pouvoirs restreints de l'Assemblée galloise provoquèrent notamment des tensions au sein de la branche galloise.

Lors des élections législatives de mai 1997, les deux principaux partis britanniques firent campagne sur des programmes opposés en matière de dévolution, les conservateurs $\mathrm{y}$ étant toujours hostiles. Cette fois-ci les travaillistes remportèrent les élections avec $44,3 \%$ des voix et 418 sièges sur 641 en Grande-Bretagne, un véritable raz-de-marée. Ils restaient toujours le parti dominant en Écosse et au pays de Galles. En revanche, les conservateurs subissaient une cuisante défaite et perdaient pour la première fois tous leurs sièges en Écosse et au pays de Galles.

\section{La concrétisation des engagements (1997-1999)}

30 Profitant de son triomphe électoral, le gouvernement travailliste publia, dès juillet 1997, deux livres blancs, donnant les grandes lignes de la législation projetée, et annonçant la date du 11 septembre pour le référendum écossais et celle du 18 septembre pour le référendum gallois.

Contrairement à 1979 , le contexte était beaucoup plus favorable au gouvernement travailliste. Premièrement, le gouvernement Blair ne se situait pas en fin de mandat et n'était ni fragilisé, ni essoufflé puisqu'il venait d'être élu avec une très forte majorité. Le contexte politique était donc plus propice même si les campagnes référendaires furent, dans un premier temps, marquées par des scandales politiques dans la région de Glasgow, puis par la mort tragique de la princesse de Galles (le 31 août) qui interrompit les campagnes pendant près d'une semaine ${ }^{47}$. Deuxièmement, les livres blancs étaient plus clairs et plus ambitieux ${ }^{48}$. Parmi les principales différences, nous pouvons noter que cette fois-ci les travaillistes proposaient à l'Écosse la création d'un Parlement (et non plus d'une Assemblée) qui disposerait de pouvoirs fiscaux, ainsi que celle d'un exécutif dirigé par un First Minister (plutôt que par un First Secretary). En ce qui concernait le mode de scrutin adopté pour l'élection des membres du Parlement / Assemblée, il s'agissait cette fois-ci d'un scrutin mixte, ce qui réduisait les craintes d'une domination des travaillistes. Enfin, si les secrétaires d'État étaient conservés, leur rôle avait été profondément modifié et limité comparé à ce que prévoyait le projet de loi de 1978. Troisièmement, les référendums précédaient cette fois-ci le vote des projets de loi aux Communes, ce qui 
évitait la mise en évidence de divisions inter- et intrapartites trop importantes tout en donnant plus de légitimité aux propositions du gouvernement lorsqu'elles seraient présentées au Parlement britannique. Enfin, les campagnes référendaires furent moins confuses et plus efficaces que celles de 1979, le camp du « oui » étant beaucoup plus uni. En Écosse, la campagne pour le «oui, oui» (Yes-yes campaign) réunissait tous les travaillistes (mis à part Tam Dalyell), les libéraux-démocrates et le SNP au sein de l'organisation transpartisane Scotland Forward face à l'organisation pour le «non, non ", Think Twice, dominée par les conservateurs, peu actifs et quelque peu démotivés en raison de leur défaite cuisante en mai 1997. Au pays de Galles, l'organisation Yes for Wales regroupait la plupart des travaillistes gallois, menés par Ron Davies, les libérauxdémocrates, le Plaid Cymru ainsi que des universitaires, face à Just Say No, organisation moins importante et regroupant les conservateurs et quelques dissidents travaillistes. Les divisions au sein de la branche galloise du Parti travailliste affaiblirent quelque peu le camp du « oui » au pays de Galles ${ }^{49}$.

Cette fois-ci, les Écossais et les Gallois soutinrent les propositions du gouvernement. En Écosse, la victoire fut spectaculaire : presque $74,3 \%$ des votants votèrent pour le retour du Parlement écossais et 63,5\% acceptèrent de lui confier des pouvoirs fiscaux. Les Gallois se montrèrent beaucoup plus timides puisque $50,3 \%$ des votants seulement se déclarèrent favorable à une Assemblée galloise alors que $49,7 \%$ s'y opposèrent. Une fois que les votes furent entérinés par le Scotland Bill et le Government of Wales Bill, qui connurent un passage assez facile au Parlement britannique, les premières élections au Parlement écossais et à l'Assemblée galloise purent être organisées le 6 mai 1999.

En Écosse, elles virent la victoire des travaillistes. Néanmoins, en raison du scrutin mixte qui avait été adopté, ils ne réussirent pas à obtenir la majorité absolue, ne recueillant que 56 sièges sur les 65 requis. Comme ils s'y attendaient, ils formèrent donc une coalition avec les libéraux-démocrates. Le Parlement écossais se réunit pour la première fois le 12 mai 1999. Dans les jours qui suivirent, Donald Dewar fut investi First Minister ; un exécutif composé de onze ministres adjoints (deputy ministers) et de onze ministres fut formé ; et un pacte de coalition Partnership for Scotland fut signé par les dirigeants travailliste (Donald Dewar) et libéral-démocrate (Jim Wallace), ces derniers s'engageant à gouverner de concert pendant les quatre années de la première législature.

$\mathrm{Au}$ pays de Galles, le Parti travailliste remporta également un très grand nombre de voix lors de ces élections ( $36,6 \%$ des voix et 28 sièges sur 60 ) mais une fois de plus, en raison du mode de scrutin, ils n'obtinrent pas la majorité absolue à l'Assemblée. Ils furent donc contraints de former une administration minoritaire dépendant des libéraux-démocrates afin de pouvoir diriger les affaires du pays. Réunie pour la première fois le 12 mai 1999, l'Assemblée élut son First Secretary Alun Michael (député de Cardiff South et Penarth), sous les pressions du gouvernement Blair.

\section{Un parti converti à la dévolution?}

Ainsi, après plus de vingt ans d'efforts, le Parti travailliste était parvenu à établir des gouvernements dévolus en Écosse et au pays de Galles. Cette victoire résultait d'un long processus au cours duquel le parti avait tenté d'éliminer les obstacles à ce projet et notamment de réduire l'opposition en son sein qui avait menacé les premiers projets de loi. Pour autant, les travaillistes n'avaient opéré aucune conversion à l'égard de la dévolution. Le parti n'envisagea jamais de mener une réflexion de fond sur cette question 
et son évolution ne fit que refléter ses préoccupations politiques du moment. La dévolution fut ainsi considérée comme un instrument permettant de freiner les progrès des partis nationalistes dans les années 1960 et 1970, de conserver le soutien de ses sympathisants travaillistes et de mettre un terme à la domination des gouvernements conservateurs en Écosse et au pays de Galles dans les années 1980 et 1990, puis, plus récemment, de moderniser l'État britannique. Ce sont donc des facteurs pragmatiques qui ont avant tout permis d'expliquer le revirement du parti à l'égard de la dévolution.

\section{NOTES}

1. Jacques LERUEZ, L'Écosse. Vieille Nation, Jeune État, Crozon : Armeline, 2000, p. 192.

2. Tam DALYELL, Devolution: The End of Britain?, London: Jonathan Cape, 1977, pp. 87-88.

3. Jacques LERUEZ, L'Écosse. Vieille Nation, Jeune État, op. cit., p. 196.

4. Kenneth O. MORGAN, Rebirth of a Nation: Wales 1880-1980, Oxford: OUP, 1981, pp. 390-391.

5. Vernon BOGDANOR, Devolution in the United Kingdom, Oxford: OUP, 1979 (second edition 2001), p. 175.

6. Kenneth O. MORGAN, Rebirth of a Nation: Wales 1880-1980, op. cit., p. 392, p. 395.

7. Dilys M. HILL, 'Devolution', p. 227 in : Anthony SELDON \& Kevin HICKSON (eds.), New Labour, Old Labour: The Wilson and Callaghan Governments 1974-79, London: Routledge, 2004.

8. Christian CIVARDI, "Le Mouvement ouvrier et la question nationale, 1886-1997 », p. 48 in: Keith DIXON (dir.), L'Autonomie écossaise : essais critiques sur une nation britannique, Grenoble : Ellug, 2001.

9. Dilys M. HILL, 'Devolution', op. cit., p. 228.

10. Ce parti allait ensuite faire campagne pour une assemblée écossaise disposant de pouvoirs dans le domaine économique, une représentation autonome de l'Écosse à Bruxelles, et la mise en œuvre de politiques socialistes. Il disparut de la scène politique écossaise après les élections législatives de 1979.

11. James Callaghan était devenu Premier ministre à la suite de la démission de Harold Wilson en mars 1976. Le gouvernement avait décidé d'inclure l'Écosse et le pays de Galles dans le même projet de loi afin d'inciter les anti-dévolutionnistes gallois à soutenir ce projet.

12. Vernon BOGDANOR, Devolution in the United Kingdom, op. cit., p. 182.

13. Kenneth O. MORGAN, Rebirth of a Nation: Wales 1880-1980, op. cit., p. 399.

14. Ce paradoxe fut notamment mis en évidence par le travailliste écossais Tam Dalyell qui représentait la circonscription écossaise de West Lothian

15. C'était par exemple le cas de Roy Jenkins (ministre de l'Intérieur), Tony Crosland, Reg Prentice et Denis Healey.

16. Frances WOOD, 'Scottish Labour in Government and Opposition: 1964-79', p. 122 in: Ian DONNACHIE et al. (eds.), Forward! Labour Politics in Scotland 1888-1988, Edinburgh: Polygin, 1989.

17. Martin WESTLAKE, Kinnock. The Biography, London: Little Brown, 2001, pp. 124-125.

18. Le Welsh Council of Labour fut rebaptisé ainsi en 1975.

19. Vernon BOGDANOR, Devolution in the United Kingdom, op. cit., p. 184.

20. Frances WOOD, 'Scottish Labour in Government and Opposition: 1964-79', op. cit., p. 127.

21. Gilles LEYDIER, La question écossaise, Rennes : Presses Universitaires de Rennes, 1998, p. 66. Tom DEVINE, The Scottish Nation: 1700-2000, London: Penguin, 1999, p. 589. 
22. Kenneth O. MORGAN, Rebirth of a Nation: Wales 1880-1980, op. cit., p. 404.

23. Gilles LEYDIER, La question écossaise, op. cit., pp. 127-128.

24. Furent notamment introduits un nouveau mode de désignation du dirigeant travailliste et le principe de resélection des députés dans les sections travaillistes des circonscriptions électorales. 25. LABOUR PARTY, 'The New Hope for Britain', Labour's Manifesto 1983, p. 31.

26. David DENVER et al., Scotland Decides: the Devolution Issue and the Scottish Referendum, London: Frank Cass, 2000, p. 29.

27. Gilles LEYDIER, La question écossaise, op. cit., p. 128.

28. ibid., pp. 131-133.

29. Jacques LERUEZ, L'Écosse. Vieille Nation, Jeune État, op. cit., pp. 249-250.

30. L'organisation transpartisane CSA avait été mise en place dès mars 1980 afin de promouvoir l'idée d'une Assemblée législative directement élue en Écosse.

31. James G. KELLAS, 'The Scottish Constitutional Convention and the Recent Institutional Developments under John Major', L'Écosse contemporaine : politique, société et culture, Revue Française de Civilisation Britannique, vol. IX, n² 2, mai 1997, pp. 41-42.

32. Ce groupe, resté silencieux depuis 1979, fut relancé sous l'impulsion de responsables du Parti travailliste, du Plaid Cymru et du Parti libéral-démocrate.

33. Depuis 1979, le nombre des organismes publics et semi-publics (quangos ou quasi autonomous non-governmental organizations), au fonctionnement non transparent et dominés par les conservateurs, s'était multiplié en Écosse. Hervé ABALAIN, «Vers une Assemblée galloise », p. 199 in : Gaïd GERARD \& Mathew GRAVES (dir.) Enjeux politiques, constitutionnels et identitaires de la dévolution, Brest : Triade, 2000.

34. LABOUR PARTY, Looking to the Future, 1990, p. 43.

35. LABOUR PARTY, 'It's time to get Britain working again', Labour's Election Manifesto, 1992, p. 23.

36. John Major, qui avait remplacé Margaret Thatcher à la tête du Parti conservateur à la suite de sa démission en 1990, remporta ces élections.

37. Mark STUART, John Smith. A Life, London: Politico's, 2005, p. 293.

38. 'Minutes of Proceedings of the Evidence of Mr Ron Davies', Commission on the Powers and Electoral Arrangements of the National Assembly for Wales, 26 September 2002. (http:// www.richardcommission.gov.uk)

39. Mark STUART, John Smith. A Life, op. cit., p. 293.

40. Jacques LERUEZ, L'Écosse. Vieille Nation, Jeune État, op. cit., p. 251.

41. John OSMOND, Welsh Europeans, Bridgend: Seren, 1995, pp. 171-172, pp. 187-188.

42. NEW LABOUR, 'Modernising Our Democracy', Because You Deserve Better, 1997, p. 11.

43. 'Minutes of Proceedings of the Evidence of Mr Ron Davies', op. cit.

44. James G. KELLAS, 'The Scottish Constitutional Convention and the Recent Institutional Developments under John Major', op. cit., p. 43.

45. John OSMOND, Welsh Europeans, op. cit., pp. 31-33.

46. Colin PILKINGTON, Devolution in Britain Today, Manchester: Manchester University Press, 2002, pp. 70 et 122 .

47. Christopher HARVIE, Scotland and Nationalism: Scottish Society and Politics, 1707 to the Present, London: Routledge, 1998, $3^{\text {rd }}$ ed., pp. 245-246.

48. Christian CIVARDI, L'Écosse contemporaine, Paris : Ellipses, 2002, p. 110.

49. Colin PILKINGTON, Devolution in Britain Today, op. cit., p. 124. 


\section{RÉSUMÉS}

À partir de 1966-67, les travaillistes ont commencé à revoir leur politique en matière de dévolution, devenant de plus en plus favorables à la mise en place d'institutions décentralisées en Écosse et au pays de Galles. Malgré l'échec des référendums de 1979, ils ont maintenu une attitude pro-dévolutionniste, mais ce ne fut qu'en 1999, après plus de vingt ans d'efforts, qu'ils parvinrent à créer un Parlement écossais et une Assemblée galloise. Quelles raisons les ont poussés à suivre cette politique ? Cette attitude répondait-elle avant tout à des considérations pragmatiques ou dogmatiques?

From 1966 onwards the Labour Party started to change its viewpoint on devolution, becoming increasingly favourable to the creation of devolved governments in Scotland and Wales. Despite the debacle of the 1979 referendums, it continued to support devolution and ultimately succeeded in creating a Scottish Parliament and a Welsh Assembly in 1999. Why did Labour follow such a policy? Did their evolution show a genuine conversion to devolution or was it a reaction to specific economic and political circumstances, i.e., to purely pragmatic reasons?

\section{AUTEUR}

\section{CARINE BERBERI}

Université de Montpellier I 